Barba-Marín, R., Sonlleva Velasco, M. \& García-Marín, N. (2018). "Presencia, participación y progreso": el aprendizaje basado en proyectos en la trayectoria de una maestra en formación. Revista Electrónica Interuniversitaria de Formación del Profesorado, 21(2), 13-25.

DOI: http://dx.doi.org/10.6018/reifop.21.2.323201

\title{
"Presencia, participación y progreso": el aprendizaje basado en proyectos en la trayectoria de una maestra en formación
}

Raúl A. Barba-Martín, Miriam Sonlleva Velasco y Noelia García-Martín

Facultad de Educación de Segovia. Universidad de Valladolid

\section{Resumen}

La formación inicial del profesorado, asentada en un enfoque curricular técnico, presenta para el docente algunas limitaciones. Superarlas, sólo es posible escuchando cómo vive el estudiante su proceso formativo. El estudio que presentamos, tiene como objetivos conocer la experiencia de una maestra sobre su formación inicial; analizar cómo sus carencias prácticas en el aula fueron compensadas con su participación en un proyecto, basado en la Investigación - Acción; y observar cómo desde esta participación, la alumna descubre el método de Aprendizaje Basado en Proyectos (ABP) y lo pone en práctica en su aula. Para llevar a cabo el estudio se analizan los diarios biográficos de la protagonista, notas de campo y una entrevista semiestructurada. El análisis de datos nos lleva a concluir, que resulta necesario ofrecer al maestro en formación el conocimiento de métodos como el $A B P$, permitirle que lleve estas experiencias a la práctica y reflexione sobre sus bondades.

\section{Palabras clave}

Aprendizaje basado en proyectos; Prácticum; investigación-acción; innovación educativa

\section{"Presence, participation and progress": project based learning in the trajectory of a teacher in training}

\begin{abstract}
Initial teacher training, based on a technical curricular approach, presents to the teacher some limitations. To get over them is only possible by listening how does the student lives
\end{abstract}

\section{Contacto:}

Raúl Alberto Barba Martín, raulalberto.barba@uva.es, Campus María Zambrano, Universidad de Valladolid, Departamento de Didáctica de la Expresión Musical, Plástica y Corporal, Plaza de la Universidad, 1, 40005, Segovia (ESP). 
his formative process. The research we present aims to know the experience of a teacher about her initial training; to analyze how their practical deficiencies in the classroom were compensated with their participation in a project, based on action-research; and to observe how from this participation, the student discovers the Project Based Learning (PBL) method and how does she put it into practice in her classroom. In order to carry out the study, biographical diaries of the teacher, field notes and a semi-structured interview are analyzed. Review of the data leads us to conclude that it is required to offer the teacher in training the knowledge of methods such as PBL, to allow them to take these experiences to practice and reflect on their advantages.

\section{Key words}

Project Based Learning; practicum; action-research; educational innovation

\section{Introducción}

La Formación Inicial del Profesorado (FIP) puede estar orientada desde diferentes enfoques curriculares -técnico, práctico y emancipador (Grundy, 1998)-. Aspectos como la burocratización, la política educativa o la brecha entre teoría y práctica han llevado tradicionalmente a la FIP a guiarse por perspectivas técnicas. Este enfoque conlleva la pérdida de un compromiso personal, cultural y político con la educación (Luengo \& Saura, 2013) e implica la asunción -y continuación- de las malas praxis (Imbernón, 2017). Las exposiciones orales teóricas son prácticas comunes del profesorado universitario e implican que los futuros docentes no adquieran las competencias necesarias para llevar a cabo su labor en el aula (Barba, 2011; Berliner, 1988; Vonk, 1988). Estas razones, hacen que la FIP no se adapte a un mundo cambiante y el alumnado únicamente adquiera conocimientos con fecha de caducidad (Marcelo, 2002). González-Calvo y Barba (2014) e Imbernón (2017) señalan la importancia de que la FIP abandone esta perspectiva técnica y adopte un enfoque curricular emancipador. La investigación se convierte así en un aspecto crucial de la FIP, haciendo de su aprendizaje un proceso crítico de autorreflexión de la realidad educativa (Cochran-Smith \& Zeichner, 2005).

Uno de los momentos clave en la FIP es su primera inserción prolongada en el aula a través del Prácticum. En esta asignatura, el estudiante empieza a poner en práctica los saberes y competencias adquiridos durante su carrera universitaria (Mayorga, Sepúlveda, Madrid, \& Gallardo, 2016). Esta experiencia constituye un momento privilegiado para que el alumno analice críticamente los modelos de enseñanza que observa (Vaillant, 2012) y a la vez se haga consciente de sus carencias formativas, sus inquietudes y sus miedos. En este momento de la FIP resulta fundamental que el maestro en formación pueda tener contacto con otros docentes experimentados, reflexionar sobre lo que está viviendo en el aula y analizar sus primeras experiencias educativas. Sin embargo, estas posibilidades están limitadas en el Prácticum debido a factores contextuales (Beyer \& Zeichner, 1990) y una FIP exenta de reflexiones críticas. Estas carencias hacen necesaria la inclusión del estudiante en procesos de Formación Permanente del Profesorado (FPP).

La participación del estudiante en proyectos de FPP, se erige como una gran oportunidad para poder desarrollar reflexiones y consolidar sus aprendizajes, pero para ello, la FPP a seguir por el alumnado en FIP debe posibilitar la reflexión sobre su experiencia educativa (Rivas, Leite, \& Prados Mejías, 2014), el contacto con docentes de diferentes centros escolares y el acercamiento a la realidad educativa (Imbernón, 2017). Desde esta perspectiva, la FPP a través de procesos de investigación-acción (I-A) se presenta como una formación propicia por sus características. 
Siguiendo esta idea, nos encontramos con experiencias formativas como la que llevó en el curso 2013/2014 a un grupo de profesores, alumnado universitario e investigadores de la Universidad de Valladolid -coordinados por José Juan Barba Martín- a presentar un Proyecto de Innovación Docente (PID) con la finalidad de transformar la docencia en las escuelas y las universidades a través del fomento de la educación inclusiva (Barba-Martín, 2014; Barba-Martín, Barba, \& Martínez Scott, 2016). Este PID -contexto de estudio en este artículo- se desarrolla bajo un enfoque de I-A. Los participantes analizan sus realidades escolares y reflexionan junto a otros compañeros acerca de sus problemáticas (Barba, 2013; Elliot, 1993). Este proceso de reflexión se produce de manera cíclica, una vez al mes, como forma de reconstruir sus prácticas educativas, para mejorarlas y transformar sus realidades escolares (Carr \& Kemmis, 1988; Elliot, 1993). Un aspecto importante de esta formación es que no existe el rol de experto que imparte conocimiento (Greenwood, 2000), sino que este se crea de forma lateral a través de las continuas reflexiones entre los participantes y la puesta en práctica de las mejoras (Hargreaves \& Shirley, 2012). Esta característica rompe con la formación tradicional que el alumnado en FIP puede estar viviendo en las aulas universitarias y le otorga un espacio para reflexionar con docentes en activo y acercarse a sus realidades educativas.

El PID, desde sus inicios, ha trabajado en la implantación de diferentes actuaciones para fomentar la inclusión -grupos interactivos, tertulias literarias dialógicas, aprendizaje cooperativo, talleres multinivel-. Actualmente, con algunas de estas actuaciones ya asentadas, una de las principales líneas de trabajo es el Aprendizaje Basado en Proyectos (ABP). Se trata de un método fundado en la resolución autónoma de problemas por parte del alumnado (Martín \& Rodríguez, 2015). Este método considera el conocimiento como un constructo por parte del alumnado (Jumaat, Tasir, Halim, \& Ashari, 2017) y tiene sus bases en los modelos constructivistas que aúnan -entre otras- las teorías de Dewey (1938), Piaget (1977) o Vygotsky (1962).

Entre las características principales del $A B P$, que hacen posible esta construcción del conocimiento, aparecen: la globalidad de un problema, la necesidad de investigación para resolverlo, el trabajo colaborativo, la unión entre la realidad y la escuela, la conexión entre alumnado, profesorado, familia y entorno, el protagonismo del alumnado o la motivación de los tutores (Balcells, 2014; Constantinou \& Nicolaou, 2018; Márquez \& Jiménez-Rodrigo, 2014; Rekalde \& García, 2015).

A pesar de ser un método en auge en las escuelas, existen pocas investigaciones en torno al $A B P$ en la formación de docentes. El principal foco de los estudios realizados hasta el momento, lo encontramos en ingeniería o TICs (Seman, Hausmann, \& Bezerra, 2018; Verbic, Keerthisinghe \& Chapman, 2017), disciplinas en las que el ABP ha sido muy utilizado desde hace años. En educación y formación del profesorado, la mayoría de las publicaciones se centran en mostrar o evaluar experiencias en el aula (Aranda \& Monleón, 2016; García, Muñoz, \& Basilotta, 2017; López, 2015; Martín \& Rodríguez, 2015; Trujillo, 2015), pero no se han encontrado trabajos que analicen los procesos formativos de los docentes y futuros docentes para poder llevarlo a cabo.

El estudio que presentamos, tiene como objetivo abrir una línea de investigación en relación a esta carencia, analizando la formación -inicial y permanente- que recibió una alumna universitaria sobre ABP y cómo esta formación le influyó en su práctica educativa. Para desarrollar este objetivo, nos centraremos en analizar la experiencia de la maestra desde tres focos de estudio: la formación inicial recibida, su participación en el PID de la Universidad de Valladolid y el desarrollo del ABP en su práctica en el aula. 


\section{Consideraciones metodológicas}

El trabajo que presentamos es de corte cualitativo y se enmarca en el método biográficonarrativo. Para su diseño, hemos tomado como punto de partida la voz y las experiencias de una maestra en formación.

Somos partidarios de la idea que afirma, que dominar varios discursos contendientes en investigación e incluir voces hasta hace escasos años silenciadas, como ha sido el colectivo de estudiantes, es el germen del cambio, especialmente si a partir del aprendizaje que genera su escucha se pueden establecer críticas entre discursos y generar conocimiento (Gee, 2005; Sierra \& Blanco, 2017).

La investigación desde esta perspectiva implica despreciar la despersonalización del alumnado y realizar una reflexión atenta y sincera a sus formas de hacer, sentir y pensar (Calvo \& Susinos, 2010). Por ello, continuaremos las siguientes líneas, haciendo una presentación de la protagonista y exponiendo cómo hemos llevado a cabo, a través de un proceso dialogado, el diseño metodológico, el análisis de datos y las conclusiones del estudio, no sin antes agradecer a la maestra la cesión de su voz.

\section{Conociendo a la maestra}

La protagonista de este estudio es una maestra española de Educación Primaria. Tiene una edad de 24 años y a su formación inicial de Grado se une la participación en varios PID, en más de una decena de cursos y jornadas de formación, así como en Congresos y otras actividades formativas. Su experiencia en el ámbito educativo se ha fundamentado en la puesta en práctica de experiencias inclusivas y metodologías activas.

\section{Diseño de la investigación}

Para la elaboración de nuestro trabajo, hemos utilizado como instrumentos para la recogida de datos, dos diarios reflexivos de la maestra, algunas notas de campo que la protagonista escribió en su etapa de formación inicial y una entrevista semiestructurada. Seguimos a autores como Bolívar, Domingo y Fernández (2001) y Blaxter, Hughes yTight (2000) para realizar una explicación más precisa acerca de estos instrumentos:

- Diarios. Utilizamos dos diarios reflexivos que la alumna redactó en los periodos de prácticas de su formación inicial de Grado, durante los cursos académicos 2015/2016 y 2016/2017. Estos informes de la vida escolar registran observaciones de las rutinas del aula, analizan sus experiencias en torno al ABP, y evidencian sentimientos y emociones vividos por la estudiante durante dichos periodos.

- Notas de campo. Esta información fue recogida por la protagonista en dos momentos diferentes: durante su formación inicial, en los periodos de prácticas (en los que la alumna transcribe anotaciones personales sobre sus observaciones en el aula); y a lo largo de su formación permanente, en las reuniones de seguimiento del PID en el que participa, realizadas de forma mensual durante los dos cursos académicos en los que realiza las prácticas en el aula.

- Entrevista a la maestra: grabada y transcrita en el mes de julio de 2017, con una duración de dos horas. El contenido de la misma trata de revelar aquellas cuestiones específicas sobre su experiencia con el $A B P$, que no quedaban suficientemente descritas a través de los documentos elaborados por la alumna.

Tras la lectura y el estudio de los datos recogidos, procedimos a la realización de un análisis temático del contenido. Para ello, fuimos extrayendo aquellos temas conectados con los focos centrales de la investigación, que sirvieron como categorías de análisis generales: 
inmersión inicial en el aula; formación inicial vs. formación permanente; y el ABP en la práctica educativa. Desde ellas fuimos trazando subcategorías de análisis, que recogen aspectos narrados de la experiencia.

Para la redacción del informe final queremos destacar, como apuntan Bolívar et al. (2001), que no nos encontramos ante textos informativos sino ante documentos narrativos, que describen una realidad a partir de formas de sentir, pensar y actuar individuales. Por ello, procederemos al análisis de resultados en base a las tres categorías de análisis generales mencionadas con anterioridad, siendo estas ordenadas según el recorrido cronológico que ha tenido la alumna durante su formación inicial. En el análisis, utilizaremos segmentos de texto, recuperados de los documentos, para recorrer junto a la protagonista la experiencia de su formación y trabajo en el aula.

A lo largo de este estudio, hemos seguido algunos principios éticos, tanto en relación a la participante como a la investigación, basándonos en las explicaciones que autores como Josselson (2007) y Moriña (2017) exponen para este tipo de estudios narrativos. En relación a la protagonista, anunciamos que su participación ha sido voluntaria; fue informada sobre los riesgos que pudieran derivarse de la investigación; obtuvimos un consentimiento informado; y hemos intentado proteger la confidencialidad de sus narraciones, eliminando cualquier información que pudiera perjudicarle. Para la realización de este trabajo, al ser la voz de la alumna el eje vertebrador del estudio, sus narraciones serán la guía de nuestro argumento para el análisis de los datos. Los extractos de texto serán expuestos sin modificaciones gramaticales ni ortográficas por parte de los investigadores, siendo estos previamente cotejados por la alumna y consentidos para su publicación.

\section{Análisis de resultados}

\section{Inmersión inicial en la práctica educativa}

Antes de comenzar el periodo de prácticas, la alumna recibe formación, a través de un seminario introductorio y comienza a redactar sus primeras reflexiones en el diario de prácticas. El análisis de estas primeras redacciones, nos permite hacer visibles algunos de los miedos e inseguridades que los docentes en formación expresan ante su inminente entrada a un centro educativo.

Hemos empezado hablando de nuestros miedos frente a este periodo tan importante en nuestra formación (...) Miedo a no congeniar con el tutor de prácticas de los centros escolares. Miedo al control de aula y posibles conflictos con los alumnos que no sepamos resolver. Miedo a que las actividades que vayamos a dirigir en el aula no funcionen y lo que es peor, que esto nos haga dudar de nuestra vocación como maestros (Diario de prácticas, 09/11/2015).

El día previo a su primera experiencia en el aula, la estudiante deja constancia de la importancia que tiene la educación en su vida personal y profesional

Mañana ya será lunes, día en el que comienzo mis primeras prácticas como maestra, un día que marcará el resto de mi vida, tanto personal como profesional. Llevo mucho tiempo esperando para poder llegar hasta aquí. Desde que en el instituto el sistema educativo me hizo fracasar. Frustrada y con ganas de cambiar la educación, remonté luchando por poder llegar a ser maestra (Diario de prácticas, 15/11/2015).

La inquietud e incertidumbre por los acontecimientos que ocurrirán el primer día de clase se hacen visibles a través de sus palabras: 
Este fin de semana y sobre todo hoy, he estado inquieta pensando en las expectativas que tengo puestas en mí misma (...) Espero estar a la altura de lo que yo misma espero de mí, aprender mucho y disfrutar este periodo tan apasionante como enriquecedor para mi formación (Diario de prácticas, 15/11/2015).

"El día esperado". Así comienza la alumna la narración de su primer día en el aula. En su relato se agolpan un cúmulo de sentimientos que pasean entre los nervios y el júbilo. La visita por las instalaciones del centro y la presentación de compañeros y compañeras ocupan sus primeras horas en el centro. Pero ese "día esperado" tiene un objetivo crucial para la maestra: conocer a su grupo de niños y niñas. El primer contacto con los menores, la distribución de estos en el aula y los materiales que utilizan llevan a la protagonista a reflexionar en su diario sobre algunos detalles que nos permiten ver síntomas de un tipo de enseñanza tradicional.

Se me ocurren soluciones como el seguimiento de metodologías cooperativas, aprovechando la distribución del aula, pues los alumnos se encuentran sentados en grupos de 4. Pero enseguida caigo en el error. No podría implantarse una metodología cooperativa en esta aula debido a la utilización de libros de texto. Las actividades del libro no dan pie a la cooperación pues son individuales (Diario de prácticas, 16/11/2015).

Los horarios, los materiales escolares y las actividades que se llevan a cabo de forma rutinaria, junto con un conocimiento más preciso del alumnado y de sus situaciones personales, ocupan las reflexiones de la alumna en los días posteriores. Tres días después del inicio de sus prácticas, la estudiante comienza sus primeras experiencias como docente:

Hoy he podido dar otras dos clases yo sola. La de educación en valores, con la misma dinámica que el día anterior, pero sin incidencias, y una clase de sociales. En el caso de la clase de sociales pienso que hubiera ido mejor si hubiera sabido de antemano de que se trataban los ejercicios, por ello he pedido a la maestra la Programación de Aula para así poderme preparar las clases (Diario de prácticas, 19/11/2015).

A través de sus palabras, podemos apreciar que los libros de texto se imponen a cualquier otro material en el aula. A pesar de ello, hay ocasiones en las que no se utiliza libro de texto, como son los Grupos Interactivos. La docente en prácticas muestra reflexiones interesantes tras participar como voluntaria en su propia clase. Su rol de docente, le provoca dificultades para la dinamización, ya que los alumnos esperan sus instrucciones más pautadas, como es habitual en clase.

En los Grupos Interactivos se requiere algo distinto, se requiere autonomía y diálogo entre iguales para resolver las actividades planteadas, y con el maestro al frente del grupo como voluntario, esa autonomía se pierde (Diario de prácticas, 20/11/2015).

De este modo, la protagonista comienza a darse cuenta de que muchas de las conductas que se gestan en el aula coinciden con modelos tradicionales y unidireccionales de enseñanza, con los que empieza a discrepar tras algunas situaciones que no le parecen correctas:

La limpieza del cuaderno también es compatible con la libertad a la hora de ejecutar la tarea. Las instrucciones que les deberíamos de haber dado podrían haber girado en torno a la limpieza y no a la forma de representar las decenas con esos cuadrados tan pautados (Diario de prácticas, 26/11/2015).

Su presencia en la realización de exámenes también supone un punto de inflexión para la alumna, pues esta observa las problemáticas que el alumnado presenta hacia este tipo de pruebas de evaluación de sus aprendizajes. 
A mi parecer, el formato de las actividades era un poco complejo y rígido. Los niños no entendían la mayoría de los ejercicios, en parte por su poca comprensión lectora, pero también por la compleja resolución de estos (Diario de prácticas, 30/11/2015).

Pronto comienza a cuestionarse el papel que el docente tiene en el aula y cómo la utilización de metodologías tradicionales resta protagonismo a los escolares en su proceso de aprendizaje.

Creo que sería muy positivo para todos los niños que la filosofía que la Comunidad de Aprendizaje trae consigo calase más hondo, potenciando más los grupos heterogéneos de trabajo, rompiendo con los libros de texto y lanzándose hacia metodologías más participativas y dialógicas. Así la Comunidad de Aprendizaje respondería de una manera más eficaz compensando las desigualdades del contexto en el que está inmersa (Diario de prácticas, 21/12/2015).

Las vivencias en torno a este hecho, generan ciertas tensiones en la maestra en formación, que pronto percibe una desconexión entre la formación universitaria y la práctica del aula. Las narraciones de su diario, también dan cuenta de algunos problemas de formación, que la alumna comienza a presenciar en su labor educativa.

En la universidad te enseñan teorías, metodologías, contenidos, lo que hay que hacer y lo que no hay que hacer, pero no te enseñan de verdad a ser maestro. Como suele decirse, la teoría es muy bonita pero la práctica no tanto (Diario de prácticas, 10/01/2016).

\section{Aprendizaje crítico: formación inicial vs. formación permanente}

Las carencias percibidas en sus primeras experiencias en el aula, llevan a la alumna a reflexionar, junto a sus compañeros y compañeras del PID, sobre algunas problemáticas vividas en el aula. En la entrevista realizada a la protagonista, esta explica la importancia que supuso para ella la participación en este proyecto.

Desde mi entrada en el proyecto supe que interiorizar la presencia, participación y progreso de todos los estudiantes debía ser la base de mi identidad docente (Entrevista, 10/ 07/ 2017).

Los debates, las interacciones con otros docentes y la reflexión conjunta fueron potenciando en la maestra una sólida base en torno al concepto de inclusión y el descubrimiento de prácticas educativas alejadas del modelo tradicional. El conocimiento de este tipo de prácticas, permiten que la alumna empiece a cuestionarse una vez asentada en el centro, cierto tipo de actuaciones, contrarias a la inclusión.

Hoy he echado de menos la clase de compensatoria dentro del aula como la semana pasada. Pienso que es muy necesaria pues noto como los niños que acuden a apoyo cada vez se distancian más del resto de la clase (Diario de prácticas, 30/11/2015).

Sus narraciones dan cuenta de cómo la protagonista percibe los problemas que generan la aplicación de metodologías tradicionales y la influencia que estas tienen en algunos grupos de estudiantes, como el alumnado con necesidades educativas.

He constatado como con el método tradicional los alumnos van perdiendo poco a poco la atención y no se optimiza tanto el tiempo de aprendizaje (...) siguiendo esta metodología más tradicional, muchos alumnos quedarían descolgados del grupo-clase, especialmente los alumnos con necesidades específicas de apoyo educativo (Diario de prácticas, 02/12/2015). 
Tras algunos meses en el centro, la maestra termina su primer periodo de prácticas y continúa su formación universitaria. En los meses posteriores, la estudiante sigue participando de forma activa en el PID.

\section{El aprendizaje basado en proyectos en la práctica educativa}

En una de estas sesiones del PID, el grupo de trabajo empieza a reflexionar sobre la metodología de ABP, a través de la lectura y posterior charla, del libro de Juanjo Vergara Aprendo porque quiero: el Aprendizaje Basado en Proyectos paso a paso. El conocimiento de este método supone un giro importante en el discurso de la alumna sobre la práctica educativa y su forma de entender la educación.

Meses más tarde, la protagonista comienza su segundo periodo de prácticas y vuelve a reflexionar en su diario sobre cómo podría mejorar su práctica educativa conectándola con el ABP.

Desde el primer día adquirí el rol de docente que se espera en este Prácticum II, encargándome de los "proyectos" que se llevan a cabo en Ciencias. He de especificar que, de acuerdo a la bibliografía existente, no se trata de la metodología de ABP de forma pura, pues no se trabajan de manera interdisciplinar todas las áreas curriculares (Vergara, 2016) (Diario de prácticas II, semana del 1 al 3 de marzo de 2017).

Las sesiones mensuales que la alumna tiene en el PID, también le ayudan a compartir las experiencias que está viviendo en el Prácticum II y a aprender de otros maestros y maestras en activo cómo trabajar la metodología de ABP.

Otra de las ventajas que me ofrecía el PID era la de comentar mis propias experiencias en un contexto de investigación-acción. Gracias al PID he podido incrementar los aprendizajes que desde el Grado estaba adquiriendo, debatiendo mensualmente con maestros y maestras en activo sobre prácticas innovadoras e inclusivas como el $A B P$ (Entrevista, 10/ 07/ 2017).

Estas enseñanzas permiten que la alumna plantee sus propios proyectos relacionados con el ABP en el aula de prácticas.

Todo ello provocó que, durante mis prácticas, pudiera llevar a cabo las metodologías aprendidas en las jornadas de formación, desde los testimonios de los participantes del PID y de la oportunidad de observar su trabajo en las aulas (Entrevista, 10/ 07/ 2017).

Los debates con otros participantes del proyecto, le permiten vencer sus temores e inseguridades iniciales a través de procesos de reflexión, en los que la maestra va tomando conciencia de su práctica con el ABP, tras el análisis de sus propias actuaciones.

En la última reunión, narré mi experiencia intentando aproximarme a esta metodología y expuse las dificultades detectadas. Como me hicieron saber estos maestros, ellos también se replantean cada día su práctica docente, aspecto clave para mejorarla. Me hicieron ver que los errores e inseguridades eran parte de ese proceso y me dieron pautas para planificar el resto del proyecto: (a) tener claro los objetivos a conseguir y no agobiarme por la presión curricular; (b) maximizar los tiempos de participación para no caer en la metodología tradicional; (c) relacionar los contenidos curriculares planificados con los intereses de los niños para que su motivación no decaiga; (d) procurar la inclusión de los alumnos en riesgo tendiendo hacia el aprendizaje dialógico y las metodologías activas. Salí muy reconfortada de aquella reunión, habiendo aprendido que me quedaba mucho por aprender y que no estaba sola en esto, una de las virtudes de la investigación-acción (Diario de prácticas II, semana del 13 al 17 de marzo de 2017). 
Pero además, estas reflexiones llevan a la protagonista a plantear algunas propuestas en su diario y notas de campo, alternativas a los métodos educativos tradicionales.

He de destacar, que los momentos de copia en el cuaderno se hacen tediosos y aburridos. Pienso que, la elaboración de un portafolio grupal mediante actividades, realizando copias para que pudieran seguir un estudio individual en sus casas, sería muy positivo para evitar este tipo de actividades (Notas, 20/04/2017).

Sus narraciones muestran la diferencia entre este tipo de métodos y el ABP, así como los logros que la propia estudiante va consiguiendo a lo largo de su segundo periodo de prácticas:

Esta sesión ha sido de las más enriquecedoras del proyecto. En primer lugar, del cuento sobre la igualdad de género "El sueño de Lola", los alumnos extrajeron reflexiones en torno a la justicia, expresando que no era justo que Lola no se pudiera dedicar a lo que ella quería sólo por ser una chica. También surgió el tema de los derechos pues, como manifestaron los alumnos, tanto hombres como mujeres tienen los mismos. Por último, estuvimos debatiendo en torno a la diversidad en general, mencionamos la diversidad intelectual y tomamos conciencia de que lo importante es la persona. Relacionamos todos estos aprendizajes con las dos normas del centro, procedentes del Modelo Dialógico de Prevención y Resolución de Conflictos y consensuadas por toda la comunidad educativa desde la condición de Comunidad de Aprendizaje del centro (Notas, 25/04/2017).

La formación recibida en el PID facilita la corrección de sus errores en la práctica a través del aprendizaje dialógico y la I-A y a la vez mejora su autoestima y su forma de percibirse como maestra en el aula.

Gracias al PID, corregí muchos de mis errores en la aplicación del ABP y mis aprendizajes se maximizaron, aportando al grupo el testimonio de una maestra novel que se adentra en el mundo del $A B P$ en pro de la inclusión educativa (Entrevista, 10/ 07/ 2017).

Estas experiencias como docente en activo y su participación dentro del PID a lo largo de su formación inicial, hacen que la alumna termine su último periodo de prácticas siendo consciente de la importancia de la educación como práctica inclusiva y las bondades de la aplicación del método de ABP.

Por todo ello, esta experiencia ha sido inolvidable y me ha hecho crecer en lo profesional y en lo personal. He tomado conciencia de que verdaderamente soy feliz haciendo este trabajo, pues me llena la labor positiva que la escuela puede llevar a cabo para mejorar la vida de los alumnos (Diario de prácticas II, semana del 15 al 19 de mayo de 2017).

\section{Discusión y conclusiones}

Al inicio de nuestro estudio, nos planteábamos como objetivo principal conocer la experiencia formativa inicial que tiene una estudiante de Magisterio, descubrir cómo conoce el ABP y analizar cómo éste método ha influido en su práctica educativa.

A lo largo de la investigación hemos podido constatar algunas críticas que autores como Puertas (2008) o Barba (2011) ya anunciaban en sus trabajos en relación a la FIP, pues ésta no dota a los estudiantes de competencias y recursos válidos para dar clase en la escuela, lo que le conduce a los docentes en formación a una situación de angustia, miedo e 
inseguridad, como ocurre a nuestra protagonista cuando accede por primera vez al Prácticum.

El relato deja claro como la vocación por la docencia de nuestra maestra se impuso en momentos de inseguridad o dificultad en la formación inicial. Como decía Freire (1993), la educación es una cuestión de sueños y esperanzas y para nuestra alumna su pasión por la docencia era más fuerte que las dificultades. Si bien, esta pasión por la docencia puede ser insuficiente cuando las dificultades se afrontan de manera individual, la colaboración con otros docentes puede hacerla más fuerte (Barba-Martín, González-Calvo, \& Martínez-Scott, 2018). El trabajo en equipo, el diálogo reflexivo y la formación permanente se perfilan como las estrategias clave para solventar las carencias de la FIP.

El estudio también ha mostrado cómo estas carencias de la FIP, pueden ser solventadas si el docente participa en Proyectos de Formación Permanente basados en la I-A. Los datos presentados se corresponden con los que aportan investigaciones como las de Martínez (2008); Perrenoud (2010); González-Calvo y Barba (2014) o Barba-Martín, Barba y MartínezScott (2016) que ya muestran, cómo a través del análisis de la propia práctica, la reflexión con otros docentes en activo, la planificación conjunta de experiencias y la puesta en práctica, permiten dotar al docente en formación de las competencias necesarias para mejorar su práctica educativa. Sin embargo, estos estudios previos, analizan las características de la FPP a través de I-A con docentes en ejercicio. Por ello, a través de esta investigación hemos podido ampliar y corroborar como este tipo de formación es también favorable para el alumnado universitario, dotándole de un espacio de reflexión y contacto con la realidad educativa con el que no cuenta en su FIP. Este hecho nos ayuda a afirmar que este tipo de FPP es un complemento necesario a la FIP.

El trabajo mostrado ha dado cuenta de cómo nuestra protagonista, gracias a su participación en el PID, conoce el método de ABP y llega a aplicarlo en sus prácticas en el aula. La investigación nos ha ayudado a corroborar los estudios de Durán y Giné (2012) mediante los cuales analizan la formación del profesorado en educación inclusiva. Para estos autores los docentes deben aprender a transformar su rol y a reflexionar y evaluar todos los aspectos de su práctica educativa en pro de planteamientos inclusivos como hizo nuestra alumna. Esta muestra cómo, en el caso concreto del $A B P$, el docente se convierte en una pieza clave, pues su puesta en práctica le exige cuestionarse su papel en el aula, repensar la práctica educativa y comprometerse con procesos de inclusión como los expuestos en trabajos como los de López (2015), Martín y Rodríguez (2015) o García, Muñoz y Basilotta (2017). Esto es debido a que, el ABP, no solo mejora la motivación de los estudiantes, sino que ayuda a los menores a contactar con situaciones de su vida cotidiana, potencia la educación inclusiva, mejora las relaciones y el clima del aula y ayuda al alumnado a desarrollar procesos de reflexión crítica.

Para finalizar, nos gustaría remarcar algunas limitaciones del trabajo y presentar futuras líneas de investigación. Desde las primeras, queremos exponer que el estudio se encuentra restringido tanto a nivel geográfico como metodológico y sería positivo ampliar el número de casos de estudio, con el objetivo de comparar experiencias en la formación docente en relación al ABP. Los resultados de la investigación también nos animan a seguir buscando nuevas preguntas de investigación relacionadas con los objetivos del estudio. Cuestiones como cuál es la influencia que la formación permanente presenta en procesos de inclusión educativa o de evaluación; qué literatura se encuentra publicada en torno al $A B P$, tanto a nivel nacional como internacional; o qué papel ofrece el $A B P$ en las interacciones entre docentes y estudiantes son solo algunos de las potenciales líneas de trabajo que puede sugerir esta investigación. 


\section{Referencias}

Aranda Mateu, P. \& Monleón García, C. (2016). El aprendizaje basado en proyectos en el área de educación física. Actividad física y deporte: ciencia y profesión, 24, 53-66.

Balcells, M. (2014). El trabajo por proyectos: Una metodología global. Cuadernos de Pedagogía, 450, 7-13.

Barba, J.J. (2011). El desarrollo profesional de un maestro novel en la escuela rural desde una perspectiva crítica. Tesis doctoral. Universidad de Valladolid.

Barba, J.J. (2013). La investigación cualitativa en educación en los comienzos del siglo XXI. En M. Díaz y A. Giráldez (Coords.). La investigación cualitativa en educación musical (pp. 23-38). Barcelona: Graó.

Barba-Martín, R. A. (2014). La formación permanente del profesorado a través de la investigación-acción. Análisis a través del Proyecto de Innovación Docente sobre educación inclusiva en Educación Infantil. (Trabajo Fin de Máster). Segovia: Universidad de Valladolid.

Barba-Martín, R.A., Barba, J.J., \& Martínez-Scott, S. (2016). La formación continua colaborativa a través de la investigación-acción. Una forma de cambiar las prácticas de aula. Contextos Educativos, 19, 161-175

Barba-Martín, R.A., González-Calvo, G., \& Martínez-Scott, S. (2018). El papel de una maestra en la inclusión de una alumna sordociega en la comunidad educativa. Educar, 54(1), 83-99

Berliner, D.C. (1988). Implications of studies on expertise in pedagogy for teacher education and evaluation. In New Directions for Teacher Assessment, Proceeding of the 1988 ETS Invitational Conference (pp. 39-68). Princeton, NJ: Educational Testing Service.

Beyer, L.E., \& Zeichner, K.M. (1990). La educación del profesorado en el contexto cultural: Más allá de la reproducción. En T. S. Popkewitz (coord.), Formación del profesorado: Tradición, teoría, práctica (pp. 272-303). Valencia: Universitat de València.

Blaxter, L., Hughes, C., \& Tight, M. (2000). Cómo se hace una investigación. Barcelona: Gedisa.

Bolívar, A., Domingo, J., \& Fernández, M. (2001). La investigación biográfico - narrativa en educación. Enfoque y metodología. Madrid: La Muralla.

Calvo Salvador, A., \& Susinos Rada, T. (2010). Prácticas de investigación que escuchan la voz del alumnado: Mejorar la universidad indagando la experiencia. Profesorado, revista de currículum y formación del profesorado, 14, 3, 75 - 88.

Carr, W., \& Kemmis, S. (1988). Teoría crítica de la enseñanza. Barcelona: Martínez Roca.

Cochran-Smith, M., \& Zeichner, K. (Eds.) (2005). Studying teacher education: the report of the AERA panel on research and teacher education. Mahwah, NJ: Lawrence Erlbaum Associates Inc.

Constantinou, C., \& Nicolaou, S. (2018). Motivation, Challenges, Support (MCS) Cycle Model for the Development of PBL Tutors. Qualitative Research in Education, 7(1)

Dewey, J. (1938). Logic: The Theory of Inquiry. New York: Holt and Co.

Durán, D., \& Giné, C. (2012). La formación del profesorado para la educación inclusiva: Un proceso de desarrollo profesional y de mejora de los centros para atender la diversidad. Revista Latinoamericana de Educación Inclusiva, 5(2), 150-173

Elliot, J. (1993). El cambio educativo desde la investigación-acción. Madrid: Morata. 
Freire, P. (1993). Pedagogía de la Esperanza. México: Siglo XXI.

García Varcárcel, A., Muñoz Repiso, V. \& Basilotta Gómez, P. (2017). Aprendizaje Basado en Proyectos (ABP): evaluación desde la perspectiva de alumnos de Educación Primaria. Revista de Investigación Educativa, 35 (1), 113 - 131.

Gee, J.P. (2005). La ideología en los discursos. Madrid: Morata.

González-Calvo, G., \& Barba, J.J. (2014). La perspectiva autobiográfica de un docente novel sobre los aprendizajes de Educación Física en diferentes niveles educativos. Cultura, Ciencia y Deporte, 9(8), 171-181

Greenwood, D.J. (2000). De la observación a la investigación-acción participativa: una visión crítica de las prácticas antropológicas. Revista de Antropología Social, 9, 27-49

Grundy, S. (1998). Producto o praxis del currículum. Madrid: Morata.

Hargreaves, A., \& Shirley, D. (2012). La cuarta vía. El futuro prometedor del cambio educativo. Barcelona: Octaedro.

Imbernón, F. (2007). Diez ideas clave: la formación permanente del profesorado. Nuevas ideas para formar en la innovación y el cambio. Barcelona: Graó.

Imbernón, F. (2017). Ser docente en una sociedad compleja: La difícil tarea de enseñar. Barcelona: Graó.

Josselson, R. (2007). The ethical attitude in narrative research. In: J. Clandinin (Ed.), Handbook of narrative inquiry (pp. 537 - 566). Thousand Oaks, CA: Sage Publications.

Jumaat, N.F., Tasir, Z., Halim, N.D.A., \& Ashari, Z.M. (2017). Project-based learning from constructivism point of view. Advanced Science Letters, 23(8), 7904-7906

López-Torres, E. (2015). Aprendizaje Basado en Proyectos para el desarrollo de competencias profesionales del maestro: una propuesta de innovación docente desde la Didáctica de las Ciencias Sociales. Didáctica de las Ciencias Experimentales y Sociales, $29,25-41$.

Luengo, J., \& Saura, G. (2013). La performatividad en la educación. La construcción del nuevo docente y el nuevo gestor performativo. Revista lberoamericana sobre Calidad, Eficacia y Cambio en Educación, 11(3), 139-153.

Marcelo, C. (2002). La formación inicial y permanente de los educadores. En Consejo Escolar del Estado. Los educadores en la sociedad del siglo XXI (pp. 161-194). Madrid: Ministerio de Educación, Cultura y Deporte.

Márquez, E. \& Jiménez-Rodrigo, M.L. (2014). Project-based learning in virtual environments: a case study of a university teaching experience. Revista de Universidad y Sociedad del Conocimiento (RUSC), 11(1), 76-90.

Martín, A., \& Rodríguez, S. (2015). Motivación en alumnos de Primaria en aulas con metodología basada en proyectos. Revista de Estudios e Investigación en Psicología y Educación, o(01), 058-062.

Martínez, J. (2008). El olvido de la investigación-acción en el asesoramiento docente y la innovación educativa. Profesorado: Revista de currículum y formación del profesorado, 12(1), 1-10.

Mayorga-Fernández, M.J.; Sepúlveda, M.P.; Madrid-Vivar, D., \& Gallardo-Gil, M. (2017). Grado de satisfacción y utilidad profesional de las prácticas externas del alumnado 
de la Facultad de Ciencias de la Educación de la Universidad de Málaga (España). Perfiles Educativos, 39(157), 140-159.

Moriña, A. (2017). Investigar con historias de vida. Metodología biográfico - narrativa. Madrid: Narcea.

Perrenoud, P. (2010). Desarrollar la práctica reflexiva en el oficio de enseñar: profesionalización y razón pedagógica. Barcelona: Graó.

Piaget, J. (1977). The development of thought: Equilibrium of cognitive structures. New York: Viking Press.

Puertas Velarde, M.C. (2008). Relaciones interpersonales en los primeros años docentes. Actas del I Congreso Internacional sobre Profesorado Principiante e Inserción Profesional a la Docencia. Sevilla: Universidad de Sevilla.

Rekalde Rodríguez, I., \& García Vílchez, J. (2015). El aprendizaje basado en proyectos: un constante desafío. Innovación educativa, 25, 219 - 234.

Rivas, J.I., Leite, A. E., \& Prados-Mejías, E. (Coords.) (2014). Profesorado, escuela y diversidad. La realidad educativa desde una mirada narrativa. Málaga: Aljibe.

Seman, L.O., Hausmann, R., \& Bezerra, E.A. (2018). On the students' perceptions of the knowledge formation when submitted to a Project-Based Learning environment using web applications. Computers and Education, 117, 16-30.

Sierra Nieto, J.E. \& Blanco García, N. (2017). El aprendizaje de la escucha en la investigación educativa. Qualitative Research in Education, 6(3), 303-326. doi:10.17583/qre.2017.2783

Trujillo, F. (2015). Aprendizaje Basado en Proyectos. Infantil, Primaria y Secundaria. Madrid: Ministerio de Educación, Cultura y Deporte.

Vaillant, D. (2012). Formación inicial del profesor para las escuelas del mañana. Diálogo Educacional, 12(35), 167-186

Verbic, G., Keerthisinghe, C., \& Chapman, A.C. (2017). A project-based cooperative approach to teaching sustainable energy systems. IEEE Transactions on Education, 60(3), 221228.

Vergara, J. (2016). Aprendo porque quiero: el Aprendizaje Basado en Proyectos (ABP) paso a paso. Madrid: SM.

Vonk, J.H.C. (1988). Perspectives on the education and training of teachers. Barcelona: Centro Unesco de Catalunya

Vygotsky, L. (1962). Thought and language. Cambridge, MA: MIT Press. 